\title{
A Streamlined Nonlinear Path Following Kinematic Controller
}

CONFERENCE PAPER in PROCEEDINGS - IEEE INTERNATIONAL CONFERENCE ON ROBOTICS AND AUTOMATION • MAY 2015

DOI: 10.1109/ICRA.2015.7140097

CITATION

1

4 AUTHORS, INCLUDING:

Jesús Manuel de la Cruz

Complutense University of Madrid

169 PUBLICATIONS 1,543 CITATIONS

SEE PROFILE

Joaquín Aranda Almansa

National Distance Education University

125 PUBLICATIONS 458 CITATIONS

SEE PROFILE
READS

89
Jose Antonio Lopez-Orozco

Complutense University of Madrid

56 PUBLICATIONS 258 CITATIONS

SEE PROFILE 


\title{
A Streamlined Nonlinear Path Following Kinematic Controller*
}

\author{
J. M. de la Cruz ${ }^{1}$, J. A. Lopez-Orozco ${ }^{2}$, E. Besada-Portas ${ }^{3}$, J. Aranda-Almansa ${ }^{4}$
}

\begin{abstract}
This paper presents a new nonlinear pathfollowing guidance method for autonomous vehicles, which integrates two guidance laws that have given good results independently in applications in autonomous ground, marine, and air vehicles. This new technique retains the best aspects of its two supporting methods, whereas it overcomes some of their drawbacks. It uses the control law by Park et al. to command the vehicle position towards a reference point. However, the position of the reference point is controlled in a different way: instead of being calculated to stay at a fixed distance forward of the vehicle, we use a strategy, inspired in the works by other authors, that controls the speed of the reference point to maintain its position at the given distance. This change does not increment the number of parameters to tune the algorithm and makes it applicable to any initial conditions and parameterized paths. The paper also analyzes the stability of this new nonlinear guidance control law and shows its effectiveness under different simulations.
\end{abstract}

\section{INTRODUCTION}

The path following problem can be described as making a vehicle converge to and follow a path that is specified without any temporal constraint [1]. Many of the path following control algorithms that have been introduced for different types of unmanned vehicles can be classified in two different groups. In the first one, the path following loop is divided in an inner control loop and an outer guidance loop. In the second one, integrated guidance and control are designed simultaneously. In this paper algorithms of the first group are considered.

Usually the inner and outer loops are designed separately using well-established design methods for control and simple strategies for guidance. The inner loop controller stabilizes the vehicle dynamics, while the outer loop controls the vehicle kinematics and computes reference commands to the inner loop controller, providing path-following capabilities. This structure is the usual one when the vehicle comes equipped with an autopilot. If there is adequate frequency separation between the guidance and control functions, the combined scheme will perform as specified according to the two subsystems [2].

The outer guidance controller can be achieved by using a PID controller [3], [4], or other strategies based on virtual

*This work was supported by the Spanish Ministry under project DPI2013-46665-C2.

${ }^{1}$ J.M. de la Cruz is with Faculty of Physics, Complutense University of Madrid, 28040 Madrid, Spain jmcruz@ucm.es

${ }^{2}$ J.A. Lopez-Orozco is with Faculty of Physics, Complutense University of Madrid, 28040 Madrid, Spain jalopez@ucm.es

${ }^{3}$ E. Besada-Portas is with Faculty of Physics, Complutense University of Madrid, 28040 Madrid, Spain ebesada@ucm.es

${ }^{4} \mathrm{~J}$. Aranda-Almansa is with Faculty of Computer Science, UNED, 28040 Madrid, Spain jaranda@dia.uned.es points tracking, line of sight guidance or virtual fields. Within the virtual point trackers, the pioneering work in [5], [6] considers a path parameterized by its arc length and a strategy of projecting the position of a wheeled mobile robot (WMR) onto the path. In that way, there is always an imaginary virtual point on the position of the path which is closest to the real WMR. The virtual point is tracked by the WMR and its movement is modeled in terms of a Serret-Frenet frame, which is a frame tangential to the path at the exact point of projection. The path following problem is then solved in the error space of this frame, calculating the desired heading angle as a function of the cross-track error. This work was extended to unmanned aerial vehicles with full account of its dynamics in [7], to autonomous surface vehicles in [8] and to autonomous underwater vehicles in [9].

However, the previous approach has the shortcoming of having singularities when the distance to the path is not well defined. This restriction is completely overcome for WMRs in [10], [11] by controlling explicitly the progression rate of the virtual point along the path. The algorithm was extended to underwater vehicles in [12], [13], to surface vehicles in [14] and to 3D unmanned aerial vehicles in [15].

Related methods are line-of-sight (LOS) guidance algorithms. These algorithms compute the desired heading angle on the basis of a velocity-path relative angle of the vehicle with respect to a virtual point located at a given distance, not necessarily constant, further ahead along the path or along the path tangential. They have been used for under actuated 3-DOF surface vessels in [2], [16]-[24], for underactuated underwater vehicles moving in 3-D space in [8], [25]-[27], and for UAVs in [28].

Other methods use a LOS guidance law related to algorithms originally developed for missile guidance, introduced by [29] for guidance of WMRs and adopted in [30], [31] for UAVs. This approach maintains the virtual target on the desired path at a given constant distance forward of the vehicle, and generates a lateral acceleration command according to the direction of the virtual target, relative to the vehicle's velocity. Furthermore, it uses the instantaneous ground speed and compensates naturally for wind. It is very attractive because of its simplicity; it approximates a proportional derivative controller when following straightline paths, and it has an element of anticipation of the desired flight path, enabling tight tracking of curved flight trajectories. The extension of this method presented in [32] makes it more operable in real systems.

An additional alternative involving a moving point on the path is presented in [33], where the motion of the reference point is coupled to the motion of the vehicle by a 
fictitious mechanical link. The method is applicable to 3-D smooth defined paths. However, the guidance law imposes constraints on the initial position and on the velocity of the vehicle, and it cannot be applied when the vehicle has a direction normal to that of the reference point.

A different kind of approach to guidance is based on the concept of vector fields [34], [35], constructed surrounding the path to be followed. This method uses a sliding mode approach to design the control signal, provides asymptotic following for paths composed of straight lines and arcs in the presence of wind disturbances, and switches on and off the fields in multi-segment paths. However, building fields that fit generally defined paths is difficult, and its global convergence proof does not consider any limits on the turning capability of the vehicle.

In this paper, we present a new guidance law in $2 \mathrm{D}$ that merges the method in [30], [31] with that in [10], [13]. As in [30], [31], we consider that a reference point is on the desired path and that the angular rotation rate of the vehicle is generated according to the direction of the reference point, relative to the vehicle's velocity. However, our method commands the speed of the reference point (adopting an strategy inspired by [10], [13]), instead of maintaining the reference point at a constant distance forward of the vehicle (as in [30], [31]). Moreover, our method controls the reference point speed to maintain it at any given constant distance forward to the vehicle, extending the strategy in [10], [13] for distance different to zero. Thus, our approach eliminates some drawbacks of its supporting strategies, while retaining the simplicity and advantages of the method given in [30], [31]. Although it has two tuning parameters (two less than [10], [13] and one more than [30], [31]), one of them can be tuned automatically from the curvature of the path, the speed of the vehicle, and the length of the constant distance to the target.

The organization of the paper is as follows. Section II describes the proposed algorithm. Section III analyses the behavior of the method when following circular path and a straight line, determining their equilibrium points and stability. Section IV compares the proposed algorithm with other related laws. Simulation results are given in section V and the conclusions are drawn in Section VI.

\section{GUIDANCE LAW}

We consider a vehicle with position $Q(t)$ moving with ground speed $V$, velocity vector $\vec{V}$, heading angle $\psi_{V}$ and commanded inertial rotation rate $\dot{\psi}_{V}(t)$ (see Fig. 1). Besides, we consider a Serret-Frenet frame (SF) at the reference point $P(t)$ on the path, which moves with it. The abscissa of the frame is tangential to the path and the ordinate is perpendicular to it. Further, $s$ represents the distance of $P$ along the path; $s_{1}$ the along-track distance error or abscissa of $Q$ in the SF frame; $y_{1}$ the cross-track distance error or ordinate of $Q$ in the SF frame; $\vec{V}_{T}$ the velocity vector, that is tangent to the path, $\psi_{T}$ its heading angle, and $\dot{s}$ the speed; and $L_{1}$ the line segment defined from $Q$ to $P$. The angles created from line $L_{1}$ to the vehicle velocity vector

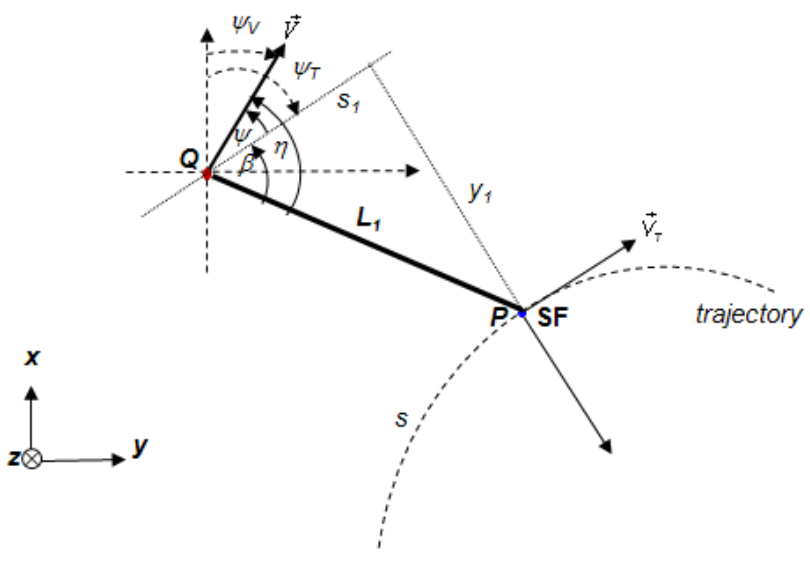

Fig. 1. UAV model

and the reference point velocity vector are denoted $\eta$ and $\beta$, respectively. The relative heading angle is defined as $\psi=\psi_{V}-\psi_{T}$. Then,

$$
\eta=\beta+\psi
$$

All the angles are clockwise positive; $\beta, \eta$ and $\psi$ are defined in the interval $[-\pi, \pi]$.

The inertial angular rotation rate of the target is

$$
\dot{\psi}_{T}=\kappa(s) \dot{s}
$$

where $\kappa(s)=1 / \rho(s)$ is the curvature of the path, and $\rho(s)$ its radius. When expressed in the SF frame, the kinematic equation of the vehicle, already presented in [10] and [13], are:

$$
\begin{gathered}
\dot{s}_{1}=-\dot{s}\left(1-y_{1} \kappa(s)\right)+V \cos \psi \\
\dot{y}_{1}=-\kappa(s) \dot{s} s_{1}+V \sin \psi \\
\dot{\psi}=\dot{\psi_{V}}-\kappa(s) \dot{s}
\end{gathered}
$$

The guidance objective is to drive the vehicle to the path and to make it follow the moving target at a given distance $L>0$. To this end, we propose the following nonlinear control law, where (6) gives the commanded angular rate for the vehicle and (7) the speed of the Serret-Frenet frame:

$$
\begin{gathered}
\dot{\psi}_{V}=\left\{\begin{array}{cc}
-\frac{2 V}{L} \sin (\eta), & |\eta| \leq \frac{\pi}{2} \\
-\frac{2 V}{L} \operatorname{sign}(\eta), & |\eta|>\frac{\pi}{2}
\end{array}\right. \\
\dot{s}=V \cos \psi+K\left(s_{1}+L\right) .
\end{gathered}
$$

This control law, which will be compared with others in Section IV, allows the tracking of the transition between a straight path and a circle, and converging exactly to paths of other shapes. Equation (6) tries to bring the cross-track error to zero, using the maximum angular rate for $|\eta| \geq 90 \mathrm{deg}$. The objective of (7) is to make the vehicle follow the moving reference point with a constant along-track error $L$. The first term of the right hand side provides the reference point with a speed that equals that of the vehicle tangentially to the path, and the second term tries to bring the reference point to a given distance in the path direction. $K$ is a positive constant 


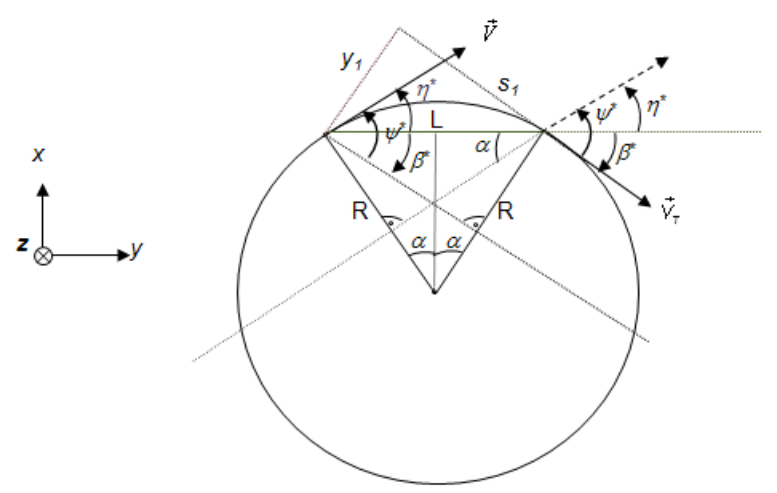

Fig. 2. Geometry of the stationary condition for a circular path

that, as Section III will show, is determined by $V, L$ and the curvature of the path. The advantages of using this type of nonlinear control law instead of linear ones, for instances PID type controllers, can be seen in [30].

\section{ANALYSIS OF THE CIRCULAR AND STRAIGHT-LINE PATH FOLLOWING}

In this section we analyze the behavior and stability of our method when following a circular path and, as a particular case, when following a straight path. We assume that the vehicle is an ideal particle so that the inner-loop control system responds instantaneously and exactly to the commanded angular rate.

If we consider a circular path of radius $R=\kappa(s)^{-1}$, the system dynamics (3-7), after substituting (6) into (5), become

$$
\begin{gathered}
\dot{s}_{1}=-\dot{s}\left(1-y_{1} / R\right)+V \cos \psi \\
\dot{y}_{1}=-\dot{s} s_{1} / R+V \sin \psi \\
\dot{\psi}=\left\{\begin{array}{l}
-\frac{2 V}{L} \sin (\eta)-\frac{V}{R}, \quad|\eta| \leq \frac{\pi}{2} \\
-\frac{2 V}{L} \operatorname{sign}(\eta)-\frac{V}{R}, \quad|\eta|>\frac{\pi}{2}
\end{array}\right. \\
\dot{s}=V \cos \psi+K\left(s_{1}+L\right) .
\end{gathered}
$$

\section{A. Stationary points}

If the vehicle and the reference point are going to follow a circle of radius $R$ with the same speed $V$, then at the stationary state $\dot{s}_{1}=0, \dot{y}_{1}=0, \dot{\psi}=0, \dot{s}=V$, the solution of the system in (8-11) and the angular relation in (1) become

$$
\begin{gathered}
s_{1}^{*}=R \sin \psi^{*} \\
y_{1}^{*}=R\left(1-\cos \psi^{*}\right) \\
\cos \psi^{*}=1-\frac{K}{V}\left(s_{1}^{*}+L\right) \\
\sin \eta^{*}=-\frac{L}{2 R}, \quad L<2 R \\
\beta^{*}=\eta^{*}-\psi^{*} .
\end{gathered}
$$

The values of $V, K, L$ and $R$ determine the relative course angle $\psi^{*}$ and through (12-13) the distance $L_{1}=\sqrt{s_{1}^{* 2}+y_{1}^{* 2}}$

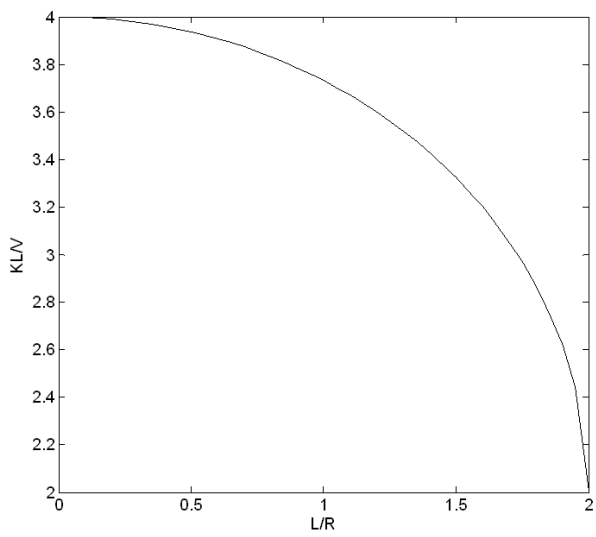

Fig. 3. Relation between $L / R$ and the non dimensional quantity $K L / V$.

of the vehicle to the target. If this distance equals $L$, then, according to Fig. 2,

$$
\begin{gathered}
\sin \beta^{*}=\sin \alpha=\frac{L}{2 R} \\
\eta^{*}=-\beta^{*} \\
\psi^{*}=-2 \beta^{*} .
\end{gathered}
$$

Besides, at the stationary point (see Fig. 2)

$$
s_{1}^{*}=-L \cos \beta^{*}
$$

and the substitution of (20) into (14) yields

$$
\frac{K L}{V}=\frac{1-\cos 2 \beta^{*}}{1-\cos \beta^{*}} \text {. }
$$

Expressions (21) and (17) show that the dimensionless quantity $K L / V$ is a function of the relation $L / R$, which is represented in Fig. 3 at the stationary condition. Therefore, the stationary point depends only on $L$ and $R$ and not on the speed of the vehicle. Moreover, although the control law in (6)-(7) has two parameters ( $L$ and $K$ ), relation (21) gives a constraint that determines $K$ adaptively as a function of the actual curvature of the path, the actual ground speed of the vehicle, and the chosen $L$.

For the special case of following a straight-line desired path, $R=\infty$, the stationary point is

$$
s_{1}^{*}=-L, y_{1}^{*}=0, \psi^{*}=0, \eta^{*}=0, \dot{s}=V,
$$

and the dynamics of the along-track error is given by

$$
\dot{s}_{1}=-K\left(s_{1}+L\right) .
$$

So, the along-track error converges to $-L$ with a time constant of $1 / K$. By linearizing the equations of the crosstrack error and course error about the equilibrium point, a second order system is obtained whose dynamics equals that of [31]. This second order system has a damping ratio of $\zeta=$ $1 / \sqrt{2}$ and an undamped natural frequency of $\omega_{n}=\sqrt{2} V / L$. In addition, from (21), $K L / V=4$, what yields

$$
K=2 \sqrt{2} \omega_{n} .
$$




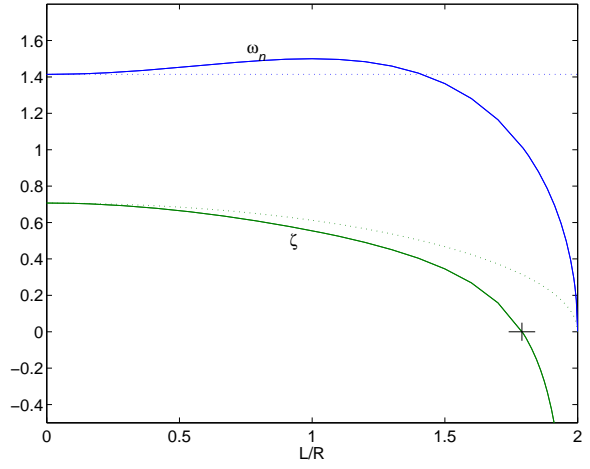

Fig. 4. The solid curves show the undamped natural frequency $\omega_{n}$ (in blue) and damping ratio $\zeta$ (in green) of the second order characteristic equation of the linearized system for different values of relation $\mathrm{L} / \mathrm{R}$. The dotted curves show the corresponding values obtained in [31].

\section{B. Stability of the stationary points}

In order to prove the stability, equations (8)-(11) can be written (see appendix for details) in term of the angles $\beta$ and $\psi$ as follows

$$
\begin{aligned}
& \frac{L}{V} \dot{\beta}=\left(\cos \psi+\frac{K L}{V}(1-\cos \beta)\right)\left(\frac{L}{R}-\sin \beta\right)+\sin (\psi+\beta) \\
& \frac{L}{V} \dot{\psi}=\left\{\begin{array}{c}
-2 \sin (\beta+\psi)-\frac{L}{R}\left(\cos \psi+\frac{K L}{V}(1-\cos \beta)\right), \\
|\beta+\psi| \leq \frac{\pi}{2} \\
-2 \operatorname{sign}(\beta+\psi)-\frac{L}{R}\left(\cos \psi+\frac{K L}{V}(1-\cos \beta)\right), \\
|\beta+\psi|>\frac{\pi}{2}
\end{array}\right.
\end{aligned}
$$

with domain

$$
Q=\{(\beta, \psi): \beta, \psi \in[-\pi, \pi]\}
$$

We can simplify (22)-(23) using a normalized time variable as $\tau=t V / L$, where one unit of normalized time is equivalent to the actual time it takes the vehicle to move distance $L$.

By the Hartman-Grobman theorem, if the linearization of the system has a hyperbolic equilibrium point, an open set of the equilibrium point exists where the linearized system has the same phase portrait as the original [36], [37]. By computing the Jacobian matrix for (22)-(23) at the equilibrium point, we determine the associated linearized system. Fig. 4 shows in solid lines the undamped natural frequency $\omega_{n}$ and the damping ratio $\zeta$ of the second order characteristic equation of the Jacobian for different values of the relation $L / R$, when using the normalized time variable. By analyzing the characteristic equation of the linearized system, it follows that this is asymptotically stable for values

$$
0 \leq L / R \leq 1.79
$$

and is unstable for $1.8 \leq L / R$. The equilibrium point is not hyperbolic when the damping ratio is zero. This occurs at $\zeta=1.791$ with an accuracy of 0.001 .

\section{Domain of attractions}

In order to analyze the domain of attractions of the equilibrium points we introduce the following theorem.

Theorem 1. Consider the autonomous system $\dot{x}=$ $f(x), x \in R^{2}$ and let $M \subseteq R^{2}$ be a compact invariant set for the system with only one equilibrium point in its interior and no equilibrium points on the boundary. Assume that for each initial condition in $M$ there is a unique solution, and that $f(x)$ has continuous partial derivatives in the interior of $M$. Let $J$ denote the Jacobian matrix of the system. Then, if the trace of $J$ is negative and the determinant of $J$ is positive at the equilibrium point, the domain of attraction is either the set $M$ or an open set $\Omega$, whose boundary is a positively invariant periodic orbit. In the latter case, the limit set of the trajectories not in $\Omega$ are periodic orbits.

Proof. The demonstration of Theorem 1 is based on the demonstration of Theorem 1.134 in [37]. From the hypothesis on the Jacobian matrix, the eigenvalues of the associated linear system have negative real parts. Therefore, the equilibrium point is asymptotically stable and the domain of attraction contains an open neighborhood of the equilibrium point. Let $\Omega$ denote the domain of attraction. If $\Omega=M$, then the demonstration is done. Otherwise, from the continuity of the solution of the differential equation, it follows that $\Omega$ is open. Moreover, it can be shown that its boundary is closed [37]. As the boundary of $\Omega$ is closed and it has no equilibrium point, by the Poincar-Bendixon theorem, it follows that the boundary is a closed orbit. Moreover, the Poincar-Bendixon theorem also tell us that the only limits of the trajectories in $M \backslash \Omega$ (the complement set of $\Omega$ in $M$ ) are closed orbits (so there exists a limit cycle in it, corollary 6.18 in [38]).

Theorem 1 tells us about the behavior when the hyperbolic equilibrium is stable. If the hyperbolic equilibrium point is unstable, then $M$ contains at least a limit cycle, see corollary 6.18 in [38].

For equations (22),(23) three different situations are found in relation to the equilibrium point (17)-(19): i) the domain of attraction of the equilibrium point is the set $M=Q$; ii) there exists an open set $\Omega \subset Q$, which is a domain of attraction of the equilibrium point with a boundary that is a limit cycle; iii) the equilibrium point is unstable and there exists a limit cycle in $Q$.

The first case is verified for $0 \leq L / R \leq 1.6$, the second is verified for $1.6<L / R \leq 1.79$, and the third is verified for $1.8 \leq L / R<2.0$. All cases can be found by simulations; however, there are certain domains of $L / R$ where case i) can be demonstrated by the isoclines method as in [31]. This occurs when $L / R=1$, whose phase plot is represented in Fig. 5. In this case, all the trajectories defined in set $Q$ converge to the stable stationary point. The cross glyph (x) are used to show those points of a trajectory that at a given time have reached the boundary and where the flow vector points outward of the definition region. Every cross glyph is related to a circle symmetric with respect to the reference axis, which shows the new initial value for the trajectory. 


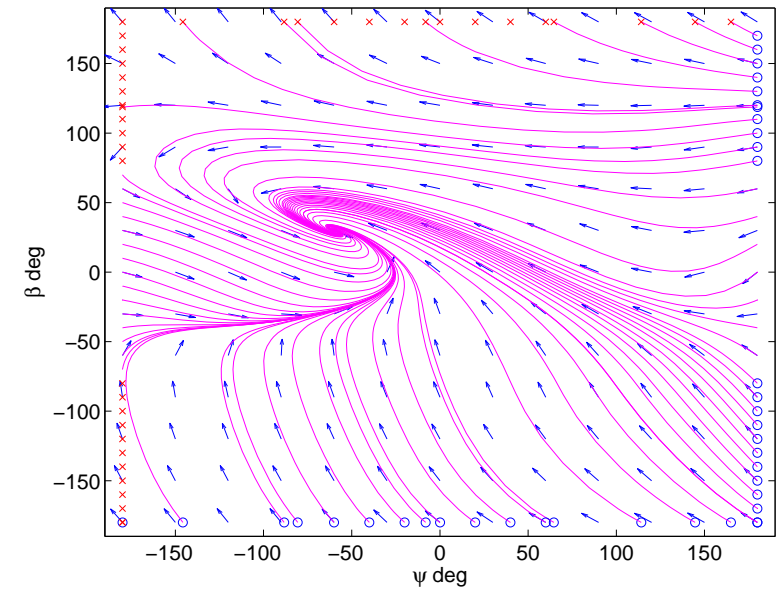

Fig. 5. The phase portrait for $L / R=1, K L / V=3.73$. All trajectories converge to the stationary point $\beta^{*}=30 \mathrm{deg}, \psi^{*}=-60 \mathrm{deg}$. Blue arrows show the flow vector.

\section{COMPARISON WITH OTHER METHODS}

In this section we relate our guidance law with other closely related algorithms and highlight the main differences and similarities.

\section{A. Comparison with [30]-[32]}

The methods in [30], [31] assume that there is always a reference point on the path at distance $L$ from the vehicle in the desired direction of travel. A nonlinear guidance law for UAVs is determined as a lateral acceleration command:

$$
a_{c m d}=2 \frac{V^{2}}{L} \sin \eta \text {. }
$$

This acceleration equals the instantaneous centripetal acceleration required to follow the circular arc that is tangent to the vehicle velocity vector, and passes through the reference point on the desired path at the constant lookahead distance $L$. This lateral acceleration is equivalent to command an instantaneous angular rotation rate

$$
\omega_{c m d}=2 \frac{V}{L} \sin \eta \text {. }
$$

Equation (26) is the law used in (6) when $|\eta| \leq \pi / 2$ for the commanded angular rate for the vehicle. The different here is that we don't consider a reference point on the path at a distance $L$ from the vehicle, but a desired distance from the vehicle to the reference point on the path. In our case, there is no need of an algorithm to find a point on the path. Given an initial position of the reference point, the evolution of this point is determined by equation (7). In that way, our law can be applied even if the distance to the path is undefined, as when the vehicle is at the center of a circle, or when it is located at a distance greater than $L$.

If the system in [30], [31] is linearized when following a circular path a second order low pass linear system is obtained for the response of the cross-track error distance, with undamped natural frequency and damping ratio

$$
\omega_{n}=\frac{\sqrt{2} V}{L}, \quad \zeta=\frac{1}{\sqrt{2}} \sqrt{1-\left(\frac{L}{2 R}\right)^{2}}
$$

for the normalized time variable. These values are represented in Fig. 4 for different values of $L / R$. In both cases, our approach and this one, the damping ratio is a function of $L / R$ and when this value tends to zero both equations tends to the value obtained for the straight-line case. The differences are not very significant when $L / R$ has values in the interval $[0,1]$, e.g., for $L / R=1$, the linearized equations of (22)-(23) give $\zeta=0.55, \quad \omega_{n}=1.50$ and equation (27) gives $\zeta=0.61, \quad \omega_{n}=1.41$. The differences are due to the different laws used for the speed of the reference point, (6) in our method and $\dot{s}=V \cos \eta / \cos \beta$ in [30], [31].

The asymptotic stability of the guidance law in [30], [31] is demonstrated for straight and circular paths, only when

$$
|\eta|<90 \mathrm{deg} \quad|\beta|<90 \mathrm{deg},
$$

even in the presence of limitations on the lateral acceleration, and it is independent of the vehicle's velocity. However, the convergence to a straight line requires that the initial crosstrack distance error is smaller than the fixed constant distance between the vehicle and the reference point. Moreover, the convergence to a circle is limited to cases where the distance is smaller than the circle radius, the reference point has to be ahead of the vehicle and the reference point will only initially move along the desired path in the forward direction.

In [32] the law of [31] is extended in several ways. The lookahead distance is computed as a linear function of the ground speed

$$
L=V T^{*}
$$

where $T^{*}$ is a specified time constant related to the roll dynamics of the UAV. In that way, the natural frequency of the linearized response of the kinematic controller is independent of ground speed. Clearly, this can be also applied to (6). In addition, [32] provides a set of rules to account for the cases where the reference point on the path is undefined, but that is not necessary with (6-7). The other rules presented in [32] can be applied to our algorithm, such as limiting the maximum value of $\eta$ in (6) when required.

Finally, we want to point out that [39] makes a detailed analysis of several guidance algorithms, including [31], which gets good results but seem to work worse than vector fields methods [34] when following a route made of circles and straight lines. A close inspection of the results shows that the routes followed by the UAVs with each algorithm are different. The longer path obtained by the algorithm in [31] is due to the method applied to compute the reference point. Our method will tackle this problem automatically, making the UAV follow a similar route as the one followed with the vector fields method.

\section{B. Comparison with [3], [13]}

The kinematic control law for path following presented in [13], which assumes that there is no side-slip, is

$$
\begin{gathered}
\dot{s}=V \cos \psi+K_{2} s_{1} \\
\dot{\psi}_{V}=\dot{\delta}-K_{1}(\psi-\delta)+\kappa(s) \dot{s}
\end{gathered}
$$


where $\delta$ is an odd function of the cross-track distance error defining the desired approach angle.

A typical choice of $\delta\left(y_{1}\right)$, used in [13], is $\delta\left(y_{1}\right)=-\psi_{a} \tanh \left(K_{\delta} y_{1}\right) \quad$ where $\psi_{a}$ is the maximum approach angle value and $K_{\delta}$ is a positive constant. An alternative approach for $\delta\left(y_{1}\right)$, motivated by the LOS algorithms [19], [26] and given in [3], is $\delta\left(y_{1}\right)=\arctan \left(-\frac{y_{1}}{\Delta}\right)$ where $\Delta$ is a constant lookahead distance along the path tangential. With these choices, either four $\left(K_{1}, K_{2}, K_{\delta}, \psi_{a}\right)$ or three $\left(K_{1}, K_{2}, \Delta\right)$ parameters need to be tuned for the kinematic control law depending on the chosen desired approach angle, instead of the only one required in our approach.

For the methods in [3], [13], the equilibrium point $\left(s_{1}, y_{1}, \psi\right)=(0,0,0)$ of system (3)-(5) when controlled with (28)-(29) is uniformly globally asymptotically and locally exponentially stable for $K_{1}>0$ and $K_{2}>0$.

\section{SIMULATIONS}

To illustrate the behavior of the proposed algorithm, we use a vehicle with the following kinematic model:

$$
\begin{aligned}
& \dot{x}=V \cos \psi_{V_{l}}+w_{x} \\
& \dot{y}=V \sin \psi_{V_{l}}+w_{y} \\
& \ddot{\psi}_{V_{l}} * \tau+\dot{\psi}_{V_{l}}=\dot{\psi}_{V}
\end{aligned}
$$

where $w_{x}$ and $w_{y}$ are the components of the wind in the north and east directions, respectively. The inner loop is modeled as a first order lag with time constant $\tau$.

In all simulations the vehicle has a constant value $V=16 \mathrm{~m} / \mathrm{s}, \tau=1 \mathrm{sec}$, and a constant wind speed of $8 \mathrm{~m} / \mathrm{s}$.

Fig. 6 shows the results when the vehicle must follow a circle in the clockwise direction and it is situated at a distance much longer than the specified distance $L$. The wind is from the East and the initial position of the reference point is $(0,150)$. The work in [32] shows that the time constant $T=L / V$ should be chosen 3 to 4 times the inner-loop dynamics to ensure satisfactory transient response. Following this rule, we use two different values of $L: L=3 * V=48 m$ and $L=2 * V=32 \mathrm{~m}$. As expected, worse transient is obtained with smaller $L$, but better following of the circle is achieved. When $L=3 * V$ the mean following error when the circle has been reached is $1.0 \mathrm{~m}$ with standard deviation of $2.7 \mathrm{~m}$, and when $L=2 * V$ the mean value is $0.5 \mathrm{~m}$ with standard deviation $1.2 \mathrm{~m}$. When applying the guidance law (6)-(7), condition $\dot{s} \geq 0$ is imposed to avoid the reference point going backward. This behavior is observed in Fig 6.b, where the reference point stand at $\dot{s}=0$ for a time waiting for the vehicle to approach it.

The example in Fig. 7 shows how the proposed method can also be used successfully to follow parameterized curves. These kinds of curves appear naturally when a UAV must operate in realistic scenarios, [40]. In this case the position of the reference point, with respect to the global reference frame, is parameterized as a third order polynomial of $s$.

$$
x(s)=\sum_{i=0}^{3} a_{i} s^{i}, \quad y(s)=\sum_{i=0}^{3} b_{i} s^{i}
$$

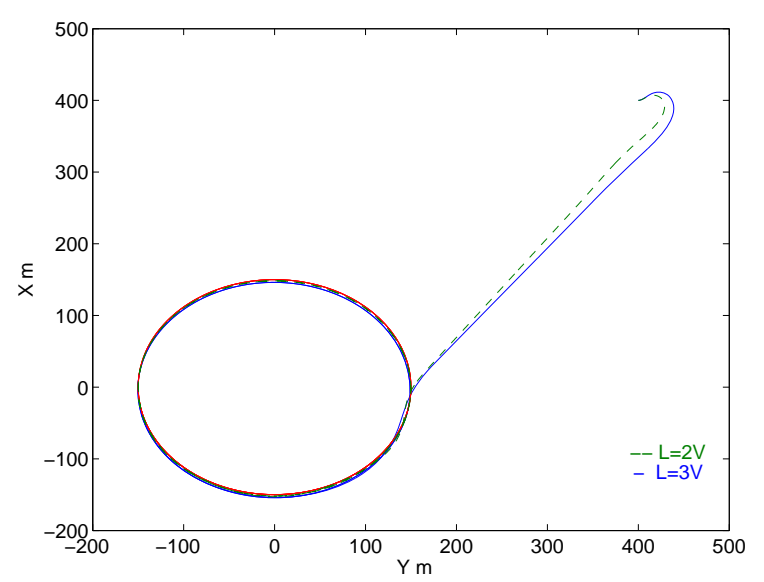

(a) Trajectory of the vehicle (green and blue) and the reference point (red)

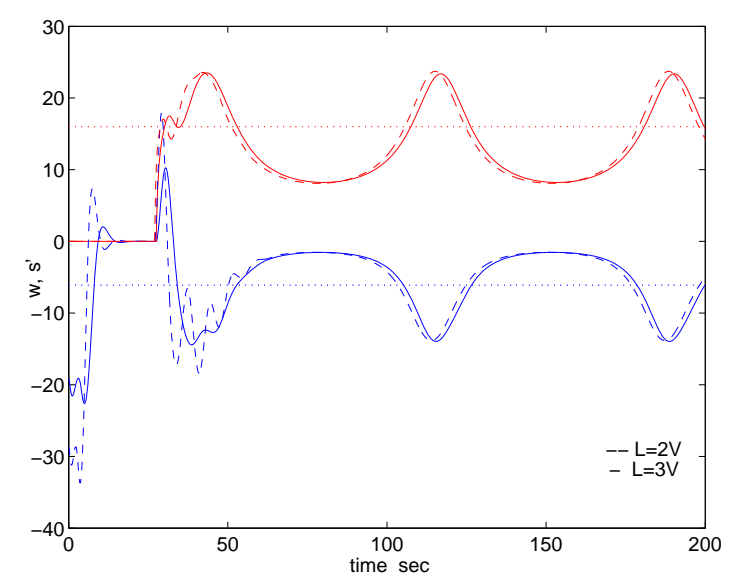

(b) Control signals: $\dot{\phi}_{V}(\mathrm{deg} / \mathrm{sec})$ in blue, and $\dot{s}(\mathrm{~m} / \mathrm{sec})$ in red

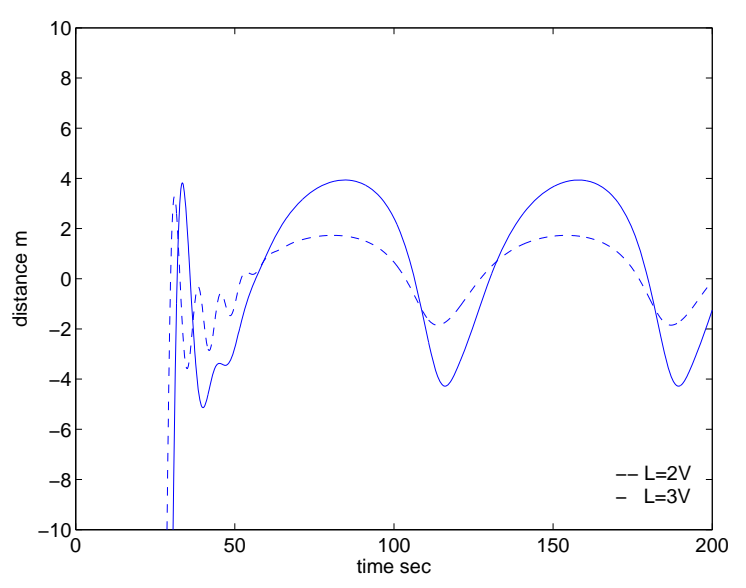

(c) Distance of the vehicle to the circle

Fig. 6. Simulation results when following a circle

where

$$
a_{0}=0, a_{1}=1.3481, a_{2}=-0.0016482, a_{3}=5.0578 \times 10^{-7}
$$$$
b_{0}=0, b_{1}=0.61188, b_{2}=0.00030765, b_{3}=-9.0729 \times 10^{-8}
$$ 
The minimum radius of curvature of the path is $111 \mathrm{~m}$. The initial position of the reference point and the vehicle are $(0,0)$ and $(0,-400)$, respectively; the initial heading of the vehicle is $0 \mathrm{rad}$ and the wind direction is $135 \mathrm{deg}$. The wind is South East. Three different values of $L$ have been considered: $L=2 \mathrm{~V}, L=4 \mathrm{~V}, L=6 \mathrm{~V}$. The maximum error occurs at the curves and have values of $0.5,1.5$ and $4 \mathrm{~m}$ respectively. Clearly, the shortest the lookahead distance, the smaller the error. However, this behavior is achieved spending higher energy values.

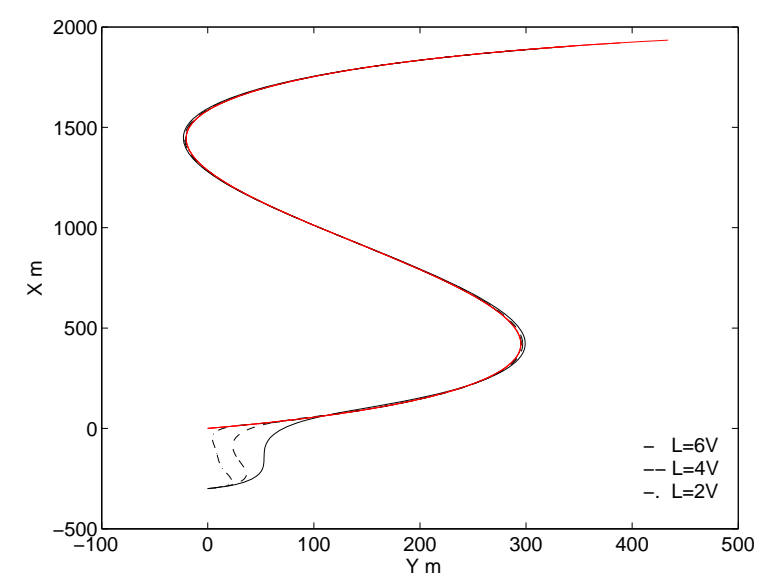

(a) Trajectory of the vehicle (black) and the reference point (red)

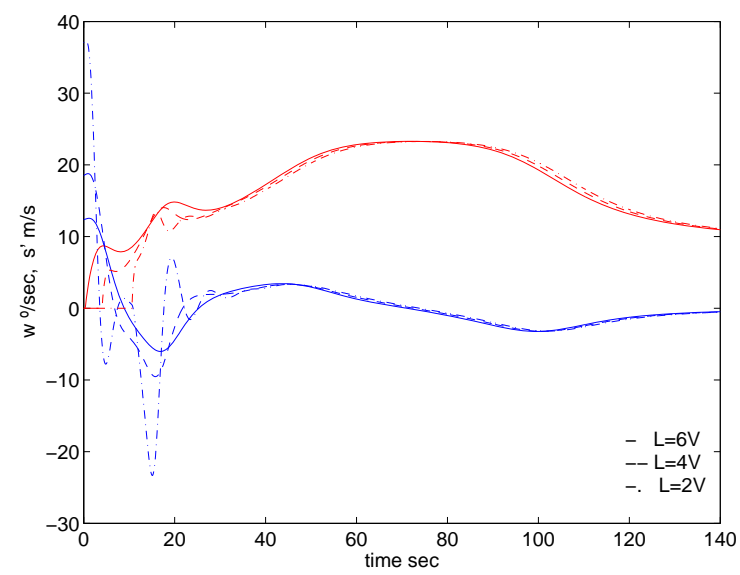

(b) Control signals: $\dot{\phi}_{V}(\mathrm{deg} / \mathrm{sec})$ in blue, and $\dot{s}(\mathrm{~m} / \mathrm{sec})$ in red

Fig. 7. Simulation results when following a parametrized curve

\section{CONCLUSIONS}

This paper presents a nonlinear path-following guidance law, which combines the basic elements of two different methods that have shown good performance in tight tracking of curved trajectories. The new method controls the speed of a moving virtual reference point on the desired path, which is coupled to the motion of the vehicle by a fixed look-ahead distance. There are two parameters in the guidance law; however, only one, the look-ahead distance, has to be tuned. The other is determined from the speed of the vehicle, the look-ahead distance, and the curvature of the path. The new method allows the vehicle to have any initial position and orientation, overcoming some of the limitations that appear in its supporting techniques. Moreover, the algorithm is well adjusted to follow parameterized paths and not only paths built from straight segments and circles.

The stability of the guidance law is demonstrated for the entire space of initial conditions and for all velocities. A limit on the values of the ratio of the look-ahead distance to the radius of the path for the asymptotic stability has been obtained.

\section{APPENDIX}

Equations (3-7) can be written in term of the angles $\beta$ and $\psi$. We derive the equations of the position of the vehicle with respect to the SF frame to obtain the derivative of angle $\beta$. We consider that the vehicle is at distance $L$ from the reference point. As can be seen in Fig. 1, the vehicle's coordinates in the SF frame are given by

$$
\begin{aligned}
& y_{1}=L \sin \beta \\
& s_{1}=-L \cos \beta
\end{aligned}
$$

From these equations, it follows that

$$
\begin{aligned}
& \dot{y}_{1}=\dot{L} \sin \beta+L \dot{\beta} \cos \beta \\
& \dot{s}_{1}=-\dot{L} \cos \beta+L \dot{\beta} \sin \beta
\end{aligned}
$$

Hence,

$$
\dot{\beta}=\frac{\dot{y}_{1}-\dot{L} \sin \beta}{L \cos \beta}=\frac{\dot{s}_{1}+\dot{L} \cos \beta}{L \sin \beta}
$$

From the second equality in (32), the following relation is derived

$$
\dot{L}=\dot{y}_{1} \sin \beta-\dot{s}_{1} \cos \beta
$$

Substituting (33) into (32) and operating, leads to

$$
\dot{\beta}=\frac{\dot{y}_{1} \cos \beta}{L}+\frac{\dot{s}_{1} \sin \beta}{L}
$$

Substituting $\dot{s}_{1}, \dot{y}_{1}$ from (3), (4) and $s_{1}, y_{1}$ from (30) leads to

$$
\dot{\beta}=\left(\kappa(s)-\frac{\sin \beta}{L}\right) \dot{s}+\frac{V}{L} \sin (\psi+\beta)
$$

Concerning the dynamics of $\psi$, substituting (1) and (6) into (5) yield

$$
\dot{\psi}= \begin{cases}-2 \frac{V}{L} \sin (\beta+\psi)-\kappa(s) \dot{s}, & |\beta+\psi| \leq \frac{\pi}{2} \\ -2 \frac{V}{L} \operatorname{sign}(\beta+\psi)-\kappa(s) \dot{s}, & |\beta+\psi|>\frac{\pi}{2}\end{cases}
$$

Now, (35), (36) and (7) describe the kinematics of the vehicle. Considering a constant curvature $\kappa(s)=R^{-1}$, (35)(36) can be written in the following non dimensional way

$$
\frac{L}{V} \dot{\beta}=\left(\cos \psi+\frac{K L}{V}(1-\cos \beta)\right)\left(\frac{L}{R}-\sin \beta\right)+\sin (\psi+\beta)
$$




$$
\frac{L}{V} \dot{\psi}=\left\{\begin{array}{l}
-2 \sin (\beta+\psi)-\frac{L}{R}\left(\cos \psi+\frac{K L}{V}(1-\cos \beta)\right) \\
|\beta+\psi| \leq \frac{\pi}{2} \\
-2 \operatorname{sign}(\beta+\psi)-\frac{L}{R}\left(\cos \psi+\frac{K L}{V}(1-\cos \beta)\right) \\
|\beta+\psi|>\frac{\pi}{2}
\end{array}\right.
$$

with domain

$$
Q=\{(\beta, \psi): \beta, \psi \in[-\pi, \pi]\} .
$$

\section{REFERENCES}

[1] A. P. Aguiar and J. P. Hespanha, "Trajectory-tracking and pathfollowing of underactuated autonomous vehicles with parametric modeling uncertainty," Automatic Control, IEEE Transactions on, vol. 52, no. 8, pp. 1362-1379, 2007.

[2] F. A. Papoulias, "Guidance and control laws for vehicle pathkeeping along curved trajectories," Applied Ocean Research, vol. 14, pp. 291$302,1992$.

[3] T. I. Fossen, Handbook of marine craft hydrodynamics and motion control. John Wiley \&amp; Sons, 2011.

[4] I. Rhee, S. Park, and C.-K. Ryoo, "A tight path following algorithm of an uas based on pid control," in SICE Annual Conference 2010, Proceedings of. IEEE, 2010, pp. 1270-1273.

[5] C. Samson, "Path following and time-varying feedback stabilization of a wheeled mobile robot," in Int. Conf. ICARCV, vol. 92, 1992.

[6] A. Micaelli and C. Samson, "Trajectory tracking for unicycle-type and two-steering-wheels mobile robots," INRIA. Sophia-Antipolis, Tech. Rep. 2097, November 1993.

[7] I. Kaminer, A. Pascoal, E. Hallberg, and C. Silvestre, "Trajectory tracking for autonomous vehicles: An integrated approach to guidance and control," Journal of Guidance, Control, and Dynamics, vol. 21, no. 1, pp. 29-38, 1998.

[8] P. Encarnacao, A. Pascoal, and M. Arcak, "Path following for autonomous marine craft," in 5th IFAC Conference on Maneuvering and Control of Marine Craft, 2000, pp. 117-22.

[9] P. Encarnacao and A. Pascoal, "3d path following for autonomous underwater vehicle," in Proc. 39 th IEEE Conference on Decision and Control. Citeseer, 2000.

[10] D. Soetanto, L. Lapierre, and A. Pascoal, "Adaptive, non-singular path-following control of dynamic wheeled robots," in Decision and Control, 2003. Proceedings. 42nd IEEE Conference on, vol. 2. IEEE, 2003, pp. 1765-1770.

[11] L. Lapierre, D. Soetanto, and A. Pascoal, "Nonsingular path following control of a unicycle in the presence of parametric modelling uncertainties," International Journal of Robust and Nonlinear Control, vol. 16, no. 10, pp. 485-503, 2006.

[12] — "Nonlinear path following with applications to the control of autonomous underwater vehicles," in Decision and Control, 2003. Proceedings. 42nd IEEE Conference on, vol. 2. IEEE, 2003, pp. 1256-1261.

[13] L. Lapierre and D. Soetanto, "Nonlinear path-following control of an auv," Ocean Engineering, vol. 34, no. 11, pp. 1734-1744, 2007.

[14] M. Bibuli, G. Bruzzone, M. Caccia, and L. Lapierre, "Path-following algorithms and experiments for an unmanned surface vehicle," Journal of Field Robotics, vol. 26, no. 8, pp. 669-688, 2009.

[15] I. Kaminer, A. Pascoal, E. Xargay, N. Hovakimyan, C. Cao, and V. Dobrokhodov, "Path following for small unmanned aerial vehicles using 11 adaptive augmentation of commercial autopilots," Journal of guidance, control, and dynamics, vol. 33, no. 2, pp. 550-564, 2010.

[16] F. A. Papoulias, "Stability considerations of guidance and control laws for autonomous underwater vehicles in the horizontal plane," in Proceedings 7th International Symposium on Unmanned Underwater Submersible Technology, Durham, New Hampshire, 1991.

[17] K. Y. Pettersen and E. Lefeber, "Way-point tracking control of ships," in IEEE Conference on Decision and Control, vol. 1. IEEE; 1998, 2001, pp. 940-945.

[18] T. I. Fossen, M. Breivik, and R. Skjetne, "Line-of-sight path following of underactuated marine craft," Proceedings of the 6th IFAC MCMC, Girona, Spain, pp. 244-249, 2003.

[19] M. Breivik and T. I. Fossen, "Path following for marine surface vessels," in OCEANS'04. MTTS/IEEE TECHNO-OCEAN'04, vol. 4. IEEE, 2004, pp. 2282-2289.
[20] E. Fredriksen and K. Y. Pettersen, "Global $\kappa$-exponential way-point maneuvering of ships: Theory and experiments," Automatica, vol. 42, no. 4, pp. 677-687, 2006.

[21] S.-R. Oh and J. Sun, "Path following of underactuated marine surface vessels using line-of-sight based model predictive control," Ocean Engineering, vol. 37, no. 2, pp. 289-295, 2010.

[22] R. Skjetne, U. Jorgensen, and A. R. Teel, "Line-of-sight path-following along regularly parametrized curves solved as a generic maneuvering problem," in Decision and Control and European Control Conference (CDC-ECC), 2011 50th IEEE Conference on. IEEE, 2011, pp. 24672474.

[23] E. Peymani and T. I. Fossen, "Motion control of marine craft using virtual positional and velocity constraints," in Control and Automation (ICCA), 2011 9th IEEE International Conference on. IEEE, 2011, pp. $410-416$.

[24] W. Caharija, K. Y. Pettersen, A. J. Sørensen, M. Candeloro, and J. T. Gravdahl, "Relative velocity control and integral line of sight for path following of autonomous surface vessels: Merging intuition with theory," Proceedings of the Institution of Mechanical Engineers, Part M: Journal of Engineering for the Maritime Environment, vol. 228, no. 2, pp. 180-191, 2014.

[25] A. J. Healey and D. Lienard, "Multivariable sliding mode control for autonomous diving and steering of unmanned underwater vehicles," Oceanic Engineering, IEEE Journal of, vol. 18, no. 3, pp. 327-339, 1993.

[26] E. Børhaug and K. Pettersen, "Los path following for underactuated underwater vehicle," Proceedings of the 7th IFAC MCMC, Lisbon, Portugal, 2006.

[27] M. Breivik and T. I. Fossen, "Guidance laws for autonomous underwater vehicles," in Underwater Vehicles, A. Inzartsev, Ed. IN-TECH, 2009, ch. 4, pp. 51-76

[28] R. Rysdyk, "Uav path following for constant line-of-sight," in 2th AIAA Unmanned Unlimited. Conf. and Workshop and Exhibit, San Diego, CA, 2003.

[29] O. Amidi and C. E. Thorpe, "Integrated mobile robot control," in Fibers' 91, Boston, MA. International Society for Optics and Photonics, 1991, pp. 504-523.

[30] S. Park, J. Deyst, and J. P. How, "A new nonlinear guidance logic for trajectory tracking," in AIAA guidance, navigation, and control conference and exhibit, 2004, pp. 1-16.

[31] — , "Performance and lyapunov stability of a nonlinear path following guidance method," Journal of Guidance, Control, and Dynamics, vol. 30, no. 6, pp. 1718-1728, 2007.

[32] R. Curry, M. Lizarraga, B. Mairs, and G. H. Elkaim, "L+ 2, an improved line of sight guidance law for uavs," in American Control Conference (ACC), 2013. IEEE, 2013, pp. 1-6.

[33] D. J. Gates, "Nonlinear path following method," Journal of guidance, control, and dynamics, vol. 33, no. 2, pp. 321-332, 2010.

[34] D. R. Nelson, D. B. Barber, T. W. McLain, and R. W. Beard, "Vector field path following for miniature air vehicles," IEEE Transactions on Robotics, vol. 23, no. 3, pp. 519-529, 2007.

[35] D. A. Lawrence, E. W. Frew, and W. J. Pisano, "Lyapunov vector fields for autonomous unmanned aircraft flight control," Journal of Guidance, Control, and Dynamics, vol. 31, no. 5, pp. 1220-1229, 2008.

[36] S. Sastry, Nonlinear systems: analysis, stability, and control. Springer New York, 1999, vol. 10.

[37] C. C. Chicone, Ordinary differential equations with applications. Springer, 1999, vol. 1.

[38] J. D. Meiss, Differential dynamical systems. Siam, 2007, vol. 14.

[39] P. Sujit, S. Saripalli, and J. Borges Sousa, "Unmanned aerial vehicle path following: A survey and analysis of algorithms for fixed-wing unmanned aerial vehicless," Control Systems, IEEE, vol. 34, no. 1, pp. 42-59, 2014.

[40] E. Besada-Portas, L. de la Torre, J. M. de la Cruz, and B. de AndrésToro, "Evolutionary trajectory planner for multiple uavs in realistic scenarios," Robotics, IEEE Transactions on, vol. 26, no. 4, pp. 619634, 2010. 